\title{
The Dawn of Systems Cancer Immunotherapy
}

\section{Guangan $\mathrm{Hu}^{*}$}

David H. Koch Institute for Integrative Cancer Research at MIT, Cambridge, USA

\section{Editorial}

Systems biology is to integrate and analyzes heterogeneous data from various platforms to broadly study the state of the biological systems using computational and statistical methods, from which hypotheses can be generated for further exploration [1]. The immune system is a complex multi-scale biological system, which comprises of diverse types of innate and adaptive immune cells and involves not only intracellular biochemical networks that regulate cell fates but also intracellular networks with various immune cells by direct contact or through the secretion of local or systemic signals, to protect against infection and eliminate disease from the host. Systems immunology is an emerging field and under the large umbrella of systems biology and provides more integrated prospective on immune system and functions of various components at different system levels [2].

The immune system has the natural capacity to detect and destroy tumor cells based on their genetic abnormalities. Immune cells can infiltrate tumors to eliminate the malignant tumor cells but the immune system often fails to prevent tumor formation and progression because tumor could avoid detection and destruction by the immune system through resisting immune mediated cell death and suppressing the immune system $[3,4]$. Cancer immunotherapy is the use of the immune system to treat cancer, which is the most rapidly advancing field of cancer treatment in the past few years. Immunotherapies either stimulate specific component of the immune system to drive an anti-tumor immune response or counteract signals in the tumor microenvironment that suppress immune responses. There have been significant achievements in immunotherapies utilizing dendritic cells, cancer vaccines, anti-tumor antibodies, adoptive T cell therapy, immune checkpoint blockade, and combinations of these strategies with other modalities such as chemotherapy or radiation therapy $[5,6]$.

However, tumor-immunity interaction and function change of immune system in the tumor environment is complex whose analysis requires a systemic view to succeed in developing efficient cancer immunotherapies. Various types of immune cells in the cancer environment play important roles in the tumor progression. Several have dual roles in cancer with antitumor or pro-tumor activity, such as macrophages, $\mathrm{NK}$ cells, and $\mathrm{CD}^{+} \mathrm{T}$ cells. The interplay between immune cells and tumor cells or among immune cells (such as regulatory $\mathrm{T}$ cells, myeloid-derived suppressor cells) is essential for the functional transition of immune cells towards to a state of tolerization, exhaustion and dysfunction $[3,4]$. Current existing immunotherapies and future directions can be generalized with educating immune cell function through adoptive cells, antibodies, bioengineering and inhibitors. However, the molecular mechanisms of the dysfunctional transition of infiltrated immune cells are largely unknown. Systemswide analysis of intracellular and intercellular factors leading to the dysfunctional transition remains a big challenge. Thus, to better design intervening strategies to educate immune system for cancer immunotherapy, it is crucial to use systems immunology approaches to investigate what keys factors leading to the dysfunctional transitions of different infiltrated immune cells in the cancer microenvironment.

Network biology is the most powerful systems biology approaches to analyze the complexity of the biological system and characterize

their mode of action [7]. Reconstructions of intracellular regulatory networks and functional networks of different types of immune cells and even intercellular networks among these immune cells are vital to identify key intracellular and intercellular factors leading to the dysfunctional transitions of tumor-infiltrated immune cells. Although there are plenty of data mining to be done currently based on highcontent data deposited in public databases and publications, more precise and specific data should be collected in the future for systems analysis in a specific cancer, such as signature genes of various types of tumor-infiltrated or tolerized immune cells, soluble factors in a tumor microenvironment, tertiary structure of immune cells in the cancer microenvironment. This analysis also requires the development of bioinformatics tools to support.

Systems cancer immunotherapy will consider utilizing multiple ways at different systems levels simultaneously by targeting various components of the immune system to be fully educated with anti-tumor activity. Systems cancer immunotherapy comes of age although it still have many challenges and obstacles to overcome in understanding the basic knowledge of immune system and its interaction with the tumor microenvironment, developing systems biology tools, designing combination strategies and evaluation strategies of treatment. In the future, a patient with a specific cancer will be treated with combinations of adoptive bioengineered immune cells, vaccines, antibodies, inhibitors and other modalities such as chemotherapy or radiation therapy to rewire the immune system to eliminate the disease.

\section{References}

1. Kitano H (2002) Systems biology: a brief overview. Science 295: 1662-1664

2. Kidd BA, Peters LA, Schadt EE, Dudley JT (2014) Unifying immunology with informatics and multiscale biology. Nat Immunol 15: 118-127.

3. Zitvogel L, Tesniere A, Kroemer G (2006) Cancer despite immunosurveillance: immunoselection and immunosubversion. Nat Rev Immunol 6: 715-727.

4. Swann JB, Smyth MJ (2007) Immune surveillance of tumors. J Clin Invest 117 1137-1146.

5. GuhaThakurta D, Sheikh NA, Meagher TC, Letarte S, Trager JB (2013) Applications of systems biology in cancer immunotherapy: from target discovery to biomarkers of clinical outcome. Expert Rev Clin Pharmacol 6: 387-401.

6. Raval RR, Sharabi AB, Walker AJ, Drake CG, Sharma P (2014) Tumor immunology and cancer immunotherapy: summary of the 2013 SITC primer. J Immunother Cancer 2: 14.

7. Vidal M, Cusick ME, Barabási AL (2011) Interactome networks and human disease. Cell 144: 986-998.

*Corresponding author: Guangan $\mathrm{Hu}$, David $\mathrm{H}$. Koch Institute for Integrative Cancer Research at MIT 500 Main Street, Cambridge, MA, USA, E-mail: gahu@mit.edu

Received October 27, 2015; Accepted October 29, 2015; Published November 04, 2015

Citation: Hu G (2015) The Dawn of Systems Cancer Immunotherapy. Arch Surg Oncol 1: e101. doi: 10.4172/2471-2671.1000e101

Copyright: (c) $2015 \mathrm{Hu} \mathrm{G}$. This is an open-access article distributed under the terms of the Creative Commons Attribution License, which permits unrestricted use, distribution, and reproduction in any medium, provided the original author and source are credited. 